\title{
DESENVOLVIMENTO E MORTALIDADE LARVAL \\ DE SPODOPTERA FRUGIPERDA EM FOLHAS DE MILHO TRATADAS COM EXTRATO AQUOSO DE FOLHAS \\ DE AZADIRACHTA INDICA ${ }^{(1)}$
}

\author{
PAULO AFONSO VIANA ${ }^{(2)}$; HÉLIO TEIXEIRA PRATES ${ }^{(2)}$
}

\begin{abstract}
RESUMO
Estudou-se o efeito do extrato aquoso de folhas de nim sobre o desenvolvimento e a mortalidade de lagartas recém-eclodidas de Spodoptera frugiperda. Para constatação do efeito de contato e de ingestão, as lagartas foram pulverizadas e as folhas de milho submersas no extrato $\left(10 \mathrm{mg} \cdot \mathrm{mL}^{-1}\right)$ em laboratório e/ou pulverizadas no campo com um pulverizador de $\mathrm{CO}_{2}$. Partes de folhas de milho tratadas foram colocadas em copos plásticos para alimentar as lagartas e trocadas a cada dois dias. Adjuvantes foram adicionados ao extrato visando melhorar sua aderência às superfícies tratadas. Os parâmetros avaliados foram a mortalidade e o desenvolvimento larval. As folhas de milho submergidas e pulverizadas com o extrato causaram elevada mortalidade $(100 \%)$ e prejudicaram o desenvolvimento das lagartas sobreviventes. Na avaliação realizada 10 dias após a aplicação, o espalhante adesivo e o óleo de soja misturados ao extrato melhoraram a eficiência deste. A mortalidade das lagartas ocorreu três dias após a aplicação do extrato e a sua pulverização diretamente sobre o inseto não prejudicou o desenvolvimento larval. O extrato aquoso de nim mostrou-se com potencial para o controle de S. frugiperda.
\end{abstract}

Palavras-chave: azadiractina, inseticida natural, Insecta, lagarta-do-cartucho.

\section{ABSTRACT \\ LARVAL DEVELOPMENT AND MORTALITY OF SPODOPTERA FRUGIPERDA FED ON CORN LEAVES TREATED WITH AQUEOUS EXTRACT FROM AZADIRACHTA INDICA LEAVES}

The effect of aqueous extracts from neem leaves and spraying adjuvants were evaluated on development and mortality of neonate $S$. frugiperda larvae. Corn leaves were dipped in the aqueous extract $\left(10 \mathrm{mg} \cdot \mathrm{mL}^{-1}\right)$ in the laboratory and/or sprayed in the field with a $\mathrm{CO}_{2}$ sprayer and placed in plastic cup for larvae rearing. The treated corn leaves were replaced every other day. Corn leaves submerged and sprayed with the extract caused high larval mortality (100\%) and showed a negative effect on the larval development. The spreading agent and soybean oil mixed with neem extract improved larval mortality at the end of the 10-day period. The aqueous extract caused lethal effect on S. frugiperda larvae after three days of the application and sprayed directly on the larvae did not inhibit the larval development. The neem aqueous extract showed good potential to be used as a control agent for S. frugiperda.

Key words: azadirachtin, natural insecticide, Insecta, fall armyworm.

( $\left.{ }^{1}\right)$ Recebido para publicação em 19 de abril de 2002 e aceito em 3 de janeiro de 2003.

$\left({ }^{2}\right)$ Embrapa Milho e Sorgo, Caixa Postal 151,35701-970 Sete Lagoas (MG).E-mail: pviana@cnpms.embrapa.br e htprates@cnpms.embrapa.br 


\section{INTRODUÇÃO}

A lagarta-do-cartucho, Spodoptera frugiperda, é considerada a mais importante praga do milho no Brasil. O seu ataque ocorre em todos os estádios do milho podendo causar perdas na produção até de 38,7\% (Williams e Davis 1990; CRUz et al. 1996). O controle dessa lagarta tem sido realizado com inseticidas sintéticos, geralmente de custo elevado, com altos riscos de toxicidade e de contaminação ambiental.

O uso de extratos de plantas pode reduzir o custo de produção da lavoura, os riscos ambientais e a dependência dos inseticidas sintéticos. A planta de nim (Azadirachta indica) tem seu centro de origem no sudeste da Ásia e devido a sua adaptação é muito comum na África tropical e subtropical, sendo encontrada também na Austrália e América. Essa planta tem mostrado atividade inseticida para várias espécies de pragas.

Os frutos são a principal fonte de azadiractina, o composto com maior ação sobre os insetos. Entretanto, a casca, as folhas e o óleo das sementes também possuem essa ação (BRUNETON, 1995). Os compostos extraídos dessa planta controlam mais de 400 espécies, incluindo insetos, nematóides, fungos, bactérias e mesmo algumas viroses (NATIONAL, 1992).

Uma revisão abrangente sobre os efeitos tóxicos do nim nos insetos foi descrita por Mordue e Nisbet (2000). Segundo Schmutterer (1990), os lepidópteros são os mais sensíveis às substâncias derivadas do nim. Esse efeito tem sido particularmente demonstrado em condições de laboratório para várias espécies (SimMONDS, 2000). BlANEY et al. (1990) relataram que 1 ppm de azadiractina reduziu a alimentação de Spodoptera littoralis em dieta artificial, quando aplicado sobre um substrato para locomoção da lagarta.

Os resultados encontrados por Adel e SEHNAL (2000) para a mesma espécie, mostraram que a mistura de óleo da semente (0,1-10 ppm de azadiractina) na dieta causou interrupção e redução na alimentação, retardamento da ecdise, morte de larvas e pupas e esterilidade dos adultos emergidos. Resultados semelhantes foram encontrados por MARTINEZ e EMDEN (2001) com cinco concentrações de azadiractina $(0,01$; 0,$05 ; 0,1 ; 0,5$ e 1 ppm) misturadas na dieta artificial dessa lagarta.

MAREDia et al. (1992) avaliaram o efeito do pó (5 e $10 \mathrm{~g}$ ) e o óleo das sementes ( $1 \mathrm{~mL}$ ) de nim misturado em cada kilograma de dietas utilizadas para alimentar seis pragas do milho. Tanto o pó como o óleo da semente reduziram a sobrevivência e o desenvolvimento de Helicoverpa zea, S. frugiperda, Diatraea saccharalis e D. grandiosella. Perez et al. (1998) utili- zaram três inseticidas derivados da semente do nim para o controle de $S$. frugiperda na cultura do milho e de Acanthoscelides obtectus em sementes armazenadas de caupi. O controle de $S$. frugiperda somente foi eficiente considerando o efeito acumulativo de três ou quatro aplicações.

Segundo Mordue e Nisbet (2000), um dos principais entraves no emprego do nim são a baixa disponibilidade de sementes e o custo elevado dos produtos derivados. Portanto, a viabilização do uso de folhas no preparo de extratos pode ser uma alternativa a outras formulações.

Embora as folhas tenham menor quantidade de ingredientes ativos, são produzidas em abundância nas condições brasileiras. Assim, o objetivo deste trabalho foi avaliar o efeito do extrato aquoso de folhas de nim sobre o desenvolvimento e mortalidade de lagartas recém-eclodidas de S. frugiperda.

\section{MATERIAL E MÉTODOS}

\subsection{Efeito do extrato aquoso de folhas de nim sobre a lagarta}

O experimento foi desenvolvido em laboratório, a $26 \pm 2{ }^{\circ} \mathrm{C}$; UR de $50 \pm 10 \%$ e fotofase de 16 horas, utilizando extrato aquoso de folhas de nim. O extrato foi preparado com folhas de nim, secas ao ar livre e à sombra, durante 10 dias. Foram moídas $150 \mathrm{~g}$ (equivalente a $10 \mathrm{~g}$ do extrato bruto) de folhas e colocadas em recipiente contendo $1 \mathrm{~L}$ de água destilada (10 $\left.\mathrm{mg} \cdot \mathrm{mL}^{-1}\right)$, deixando em repouso por 24 horas. Em seguida, o extrato foi coado em tecido de algodão de malha fina.

No laboratório, três seções de $3 \mathrm{~cm}$ de comprimento de folhas de milho foram submergidas no extrato e plantas no estádio de cinco folhas, pulverizadas no campo com um pulverizador costal pressurizado com $\mathrm{CO}_{2}$ (bico leque 80.03 e pressão de $\left.40 \mathrm{lb} / \mathrm{pol}^{2}\right)$, aplicando um volume de calda de 284 L.ha ${ }^{-1}$.

As folhas tratadas com o extrato foram imediatamente trazidas para o laboratório, colocadas em copos plásticos de $50 \mathrm{~mL}$ e utilizadas para alimentar lagartas recém-eclodidas. Um grupo dessas lagartas foi pulverizado com o extrato antes de fornecer o alimento.

Cada parcela foi composta de 25 copos ( $50 \mathrm{~mL}$ ) contendo uma lagarta por copo, arranjados em um suporte de isopor. Os tratamentos avaliados encontram-se no quadro 1. 
O delineamento experimental foi inteiramente casualizado com 25 repetições. As folhas de milho utilizadas para a alimentação das lagartas foram trocadas em dias alternados. As avaliações da mortalidade larval foram realizadas aos 3 e 10 dias após o início do experimento.

O efeito do extrato sobre o desenvolvimento das lagartas remanescentes foi avaliado aos 12 dias após o início do experimento, medindo-se o comprimento, massa e largura da cápsula cefálica do inseto.

\subsection{Efeito de adjuvantes no extrato aquoso de folhas de nim}

Esse experimento visou aumentar a eficiência do controle de $S$. frugiperda, com a adição de adjuvantes na calda do extrato aquoso de folhas de nim. As dosagens dos adjuvantes utilizados foram de $30 \mathrm{~mL}$ do espalhante adesivo $\left(\mathrm{Agral}^{\circledR}\right), 352 \mathrm{~g}$ de caseína e $352 \mathrm{~mL}$ de óleo de soja comercial $\left(\right.$ Salada $^{\circledR}$ ) em 100 L da calda.

O experimento foi desenvolvido em laboratório, a $26 \pm 2^{\circ} \mathrm{C}$ UR de $50 \pm 10 \%$ e fotofase de 16 horas, com folhas do milho pulverizadas no campo e com folhas submergidas na calda de nim, conforme descrito no primeiro experimento. Como testemunha, utilizou-se o inseticida clorpirifós (288 g.ha ${ }^{-1}$ i.a.). Cada parcela foi composta de 10 copos $(50 \mathrm{~mL})$ com uma lagarta por copo, arranjados em um suporte de isopor.

O delineamento experimental foi inteiramente casualizado com cinco repetições. As folhas de milho utilizadas para a alimentação das lagartas foram trocadas em dias alternados. As avaliações de mortalidade larval foram realizadas aos 3, 10 e 33 dias após o início do experimento. Antes que os insetos na testemunha atingissem a fase de pré-pupa (aos 14 dias), foram anotados o comprimento, a massa e a largura da cápsula cefálica.
Corrigiu-se a mortalidade larval pela fórmula de Аввотт (1925), e os resultados dos experimentos, foram submetidos à análise de variância e as médias comparadas pelo teste de Tukey $(\mathrm{P} \leq 0,05)$.

\section{RESULTADOS}

\subsection{Efeito do extrato aquoso de folhas de nim so- bre a lagarta}

A avaliação realizada aos três dias após a aplicação do extrato do nim apresentou baixa mortalidade da lagarta (Quadro 1). Aos 10 dias após a aplicação, a mortalidade variou de 12,6 a $94,4 \%$, com diferenças significativas entre os tratamentos. Os melhores tratamentos foram com as folhas submergidas, mais lagartas pulverizadas e não pulverizadas com o extrato, com $94,4 \%$ e $87,3 \%$ de mortalidade respectivamente. As lagartas, alimentadas com folhas submergidas no extrato, praticamente, pararam de se alimentar dois dias após o fornecimento do alimento e a maioria morreu aos sete dias. As lagartas sobreviventes desses tratamentos sofreram redução no seu desenvolvimento (Quadro 2). Lagartas alimentadas com folhas submergidas no extrato apresentaram comprimento médio de 5,6 $\mathrm{mm}$, massa média de 4,0 mg e a média da largura da cápsula cefálica de $0,9 \mathrm{~mm}$; lagartas alimentadas com folhas não tratadas com o extrato apresentaram comprimento médio de $24,6 \mathrm{~mm}$, massa média de 205,0 mg e a média da largura de cápsula cefálica de $2,5 \mathrm{~mm}$. Nesse experimento, praticamente, não se observou a ação de contato do extrato sobre as lagartas. A maior ação de contato foi obtida pela ingestão de folhas tratadas com o extrato. No tratamento em que a planta foi pulverizada no campo, verificou-se visualmente que a cobertura das folhas do milho com o extrato não foi uniforme, podendo ter influenciado na sua baixa efetividade.

Quadro 1. Mortalidade de lagartas de Spodoptera frugiperda em milho com extrato aquoso de folhas de nim aos 3 e 10 dias após a aplicação (DAA)

\begin{tabular}{lcc}
\hline Tratamentos $^{(1)}$ & Mortalidade & Corrigida(\%) $^{(2)}$ \\
\cline { 2 - 3 } FS e LP & 3 DAA & 10 DAA \\
FS e LNP & 13,9 n.s & 94,4 a \\
FSE e LP & 11,1 & 87,3 a \\
FP e LP & 8,4 & $12,6 \mathrm{c}$ \\
FP e LNP & 14,2 & $32,2 \mathrm{~b}$ \\
CV $(\%)$ & 6,2 & - \\
\hline
\end{tabular}

Médias seguidas da mesma letra não diferem significativamente entre si pelo teste de Tukey $(P \leq 0,05)$.

$\left.{ }^{1}\right)$ FS = folhas submersas no extrato; LP = lagartas pulverizadas; LNP = lagartas não pulverizadas; FSE = folhas sem extrato; FP = folhas pulverizadas.

$\left(^{2}\right)$ Аввотт (1925). 
Quadro 2. Média ( $\pm \mathrm{EP})$ do comprimento, massa e largura da cápsula cefálica de lagartas de Spodoptera frugiperda, tratadas com extrato aquoso de folhas de nim

\begin{tabular}{lcccc}
\hline Tratamentos $\left({ }^{1}\right)$ & $\begin{array}{c}\text { Indivíduos } \\
\text { observados }\end{array}$ & Comprimento & \multicolumn{2}{c}{ Cápsula cefálica } \\
\cline { 3 - 5 } & N. $^{\mathrm{O}}$ & $\mathrm{mm}$ & $\mathrm{mg}$ & $\mathrm{mm}$ \\
FS e LP & 11 & $6,1 \pm 1,20 \mathrm{a}$ & $6,0 \pm 2,00 \mathrm{a}$ & $0,9 \pm 0,01 \mathrm{a}$ \\
FS e LNP & 37 & $5,6 \pm 0,27 \mathrm{a}$ & $4,0 \pm 0,01 \mathrm{a}$ & $0,9 \pm 0,03 \mathrm{a}$ \\
FSE e LP & 231 & $24,7 \pm 0,24 \mathrm{c}$ & $200,0 \pm 5,00 \mathrm{c}$ & $2,4 \pm 0,02 \mathrm{c}$ \\
FSE e LNP & 262 & $24,6 \pm 0,23 \mathrm{c}$ & $205,0 \pm 4,00 \mathrm{c}$ & $2,5 \pm 0,02 \mathrm{c}$ \\
FP e LP & 181 & $20,6 \pm 0,45 \mathrm{~b}$ & $133,0 \pm 8,00 \mathrm{~b}$ & $2,1 \pm 0,03 \mathrm{~b}$ \\
\hline
\end{tabular}

Médias seguidas da mesma letra na coluna não diferem significativamente entre si pelo teste de Tukey (P $\leq 0,05)$.

$\left({ }^{1}\right)$ FS = folhas submersas no extrato; LP = lagartas pulverizadas; LNP = lagartas não pulverizadas; FSE = folhas sem extrato; FP = folhas pulverizadas.

Quadro 3. Mortalidade de lagartas de Spodoptera frugiperda alimentadas com folhas de milho tratadas com extrato aquoso de folhas de nim mais adjuvantes aos 3, 10 e 33 dias após a aplicação (DAA)

\begin{tabular}{|c|c|c|c|c|c|}
\hline \multirow{3}{*}{ Tratamentos $(1)$} & \multicolumn{5}{|c|}{ Mortalidade corrigida $(\%)^{(1)}$} \\
\hline & \multicolumn{2}{|c|}{3 DAA } & \multicolumn{2}{|c|}{10 DAA } & \multirow{2}{*}{$\begin{array}{r}33 \text { DAA } \\
\text { Folha } \\
\text { pulverizada }\end{array}$} \\
\hline & $\begin{array}{c}\text { Folha } \\
\text { pulverizada }\end{array}$ & $\begin{array}{c}\text { Folha } \\
\text { submersa }\end{array}$ & $\begin{array}{c}\text { Folha } \\
\text { pulverizada }\end{array}$ & $\begin{array}{c}\text { Folha } \\
\text { submersa }\end{array}$ & \\
\hline Extrato & 0,0 & 0,0 & $45,6 \mathrm{c}$ & 100,0 & $95,4 \mathrm{~ns}$ \\
\hline Extrato + espalhante & 0,0 & 0,0 & $69,6 \mathrm{~b}$ & 100,0 & 97,7 \\
\hline Extrato + caseína & 0,0 & 0,0 & $56,5 \mathrm{c}$ & 100,0 & 93,2 \\
\hline Extrato + óleo de soja & 0,0 & 0,0 & $71,7 \mathrm{~b}$ & 100,0 & 95,4 \\
\hline Inseticida (clorpirifós) & 100,0 & 100,0 & $100,0 \mathrm{a}$ & 100,0 & 100,0 \\
\hline $\mathrm{CV}(\%)$ & 9,27 & 10,56 & & & \\
\hline
\end{tabular}

Médias seguidas da mesma letra na coluna não diferem significativamente entre si pelo teste de Tukey $(\mathrm{P} \leq 0,05)$.

( ${ }^{1}$ ) Аввотт (1925).

\subsection{Efeito de adjuvantes no extrato aquoso de folhas de nim}

Na avaliação realizada aos três dias após a instalação do ensaio, não foi verificada mortalidade das lagartas alimentadas com folhas que foram pulverizadas ou submergidas na calda de extrato de nim (Quadro 3).

$\mathrm{Na}$ avaliação realizada aos 10 dias, no tratamento em que as folhas foram pulverizadas com o extrato no campo, a mortalidade variou de $45,6 \%$ a $71,7 \%$, apresentando diferença significativa entre os tratamentos (Tukey, $\mathrm{P} \leq 0,05$ ). No tratamento com o clorpirifós houve $100 \%$ de mortalidade. As maiores mortalidades ocorreram nos tratamentos em que se adicionou óleo de soja ou espalhante adesivo, os quais apresentaram eficiência de controle de $71,7 \%$ e $69,6 \%$ respectivamente.
O extrato de nim em água e com caseína obtiveram menor eficiência, $45,6 \%$ e 56,5\% respectivamente. Esse fato ressalta a importância de um adjuvante na aplicação desse extrato, melhorando possivelmente a distribuição e a aderência da calda sobre a folha, refletindo em maior rapidez na ação inseticida. Aos 33 dias após a aplicação, verificou-se que a eficiência de controle para todos os tratamentos com o extrato pulverizado nas folhas de milho, variou de $93,2 \%$ a $97,7 \%$. Quando as folhas foram submergidas na calda, sua superfície foi uniformemente molhada e a eficiência de controle atingiu $100 \%$ para todos os tratamentos aos 10 dias após a aplicação.

Todos os tratamentos com extrato de folhas de nim prejudicaram o desenvolvimento das lagartas, destacando-se a mistura com espalhante e óleo de soja em que foram observados os menores pesos de lagartas (Quadro 4). 
Quadro 4. Média ( \pm EP) do comprimento, massa e largura da cápsula cefálica de lagartas de Spodoptera frugiperda, alimentadas com folhas de milho tratadas com extrato aquoso de folhas de nim

\begin{tabular}{|c|c|c|c|c|}
\hline \multirow{2}{*}{ Tratamentos } & \multirow{2}{*}{$\begin{array}{l}\text { Indivíduos } \\
\text { observados }\end{array}$} & \multirow{2}{*}{ Comprimento } & \multicolumn{2}{|c|}{ cápsula cefálica } \\
\hline & & & Peso & Largura \\
\hline & N. ${ }^{o}$ & $\mathrm{~mm}$ & $\mathrm{mg}$ & $\mathrm{mm}$ \\
\hline Extrato & 19 & $11,3 \pm 1,21 \mathrm{a}$ & $29,8 \pm 0,01 \mathrm{~d}$ & $1,4 \pm 0,12 \mathrm{a}$ \\
\hline Extrato + espalhante & 7 & $10,1 \pm 1,70 \mathrm{a}$ & $19,9 \pm 0,01 \mathrm{a}$ & $1,2 \pm 0,14 \mathrm{a}$ \\
\hline Extrato + caseína & 14 & $11,9 \pm 1,02 \mathrm{a}$ & $28,6 \pm 0,01 c$ & $1,4 \pm 0,06 \mathrm{a}$ \\
\hline Extrato + óleo de soja & 7 & $10,6 \pm 1,82 \mathrm{a}$ & $21,2 \pm 0,01 b$ & $1,3 \pm 0,15 \mathrm{a}$ \\
\hline Inseticida (clorpirifós) & 0 & - & - & - \\
\hline Testemunha (água) & 44 & $33,9 \pm 0,71 b$ & $41,9 \pm 0,02 \mathrm{e}$ & $3,0 \pm 0,04 b$ \\
\hline
\end{tabular}

Médias seguidas da mesma letra na coluna não diferem significativamente entre si pelo teste de Tukey $(\mathrm{P} \leq 0,05)$.

Os extratos se destacaram em relação à testemunha, que apresentou comprimento médio de lagartas de $33,9 \mathrm{~mm}$, enquanto os extratos apresentaram valores variando de 10,1 a $11,9 \mathrm{~mm}$. A largura da cápsula cefálica das lagartas criadas em folhas que não foram pulverizadas foi de $3,0 \mathrm{~mm}$, enquanto nos extratos variou de 1,2 a 1,4 mm. Observou-se, também, que algumas espécimes de lagartas alimentadas com folhas de milho tratadas com o extrato de nim apresentaram a fase larval alongada até 33 dias (dados não apresentados).

\section{DISCUSSÃO}

Os resultados obtidos nos experimentos mostraram que o extrato aquoso de folhas de nim retardou o desenvolvimento e causou alta mortalidade nas lagartas de S. frugiperda. Segundo SCHMUtTerer (1990), a ordem Lepidoptera é o grupo de insetos mais sensível ao efeito de regulador de crescimento dos derivados do nim. Esse efeito pode resultar em vários defeitos morfogenéticos e pode causar a morte dos insetos, dependendo da concentração do produto.

A azadiractina tem mostrado efeito repelente, tóxico, regulador do crescimento e da metamorfose dos insetos, afetando a biologia, oviposição, viabilidade dos ovos e causando deterrência alimentar (SChMutTerer, 1988, 1990; Mordue e BlackWell, 1993; Neves e Nogueira, 1996). Mordue et al. (1998) demonstraram que a deterrência alimentar, associadas a alterações no desenvolvimento e na ecdise, são as principais causas da mortalidade em insetos polífagos como $S$. littoralis, $S$. frugiperda e Schistocerca gregaria. A maioria das pesquisas desenvolvidas com o nim tem sido oriundas de produtos derivados de sementes trituradas (pó) e de óleo das sementes (TRINDADE et al., 2000; ViÑUELA et al., 2000; TORRES et. al., 2001).
O efeito desses produtos foi demonstrado por ViÑuela et al. (2000) para lagartas de S. exigua que, após se alimentarem de folhas tratadas com esses produtos, pararam a alimentação, apresentaram o período de ecdise estendido, ocorrendo ainda aumento da mortalidade larval e pupal e esterilização dos adultos. Mordue et al. (1998) demonstraram que a deterrência alimentar é a principal causa da mortalidade em insetos polífagos como $S$. littoralis, $S$. frugiperda e Schistocerca gregaria. Esses resultados obtidos com a semente do nim mostram concordância com o extrato aquoso de folhas utilizado nesse trabalho em relação à redução no desenvolvimento e na mortalidade larval de S. frugiperda.

A grande vantagem desse trabalho com a utilização das folhas em relação ao uso de sementes é a facilidade de se obter grande quantidade de folhas para o preparo do extrato aquoso, tendo em vista que a produção de sementes em algumas regiões do Brasil é, muitas vezes, reduzida e o processamento para obtenção do óleo na propriedade é demorado, dificultando seu uso pelo agricultor.

Outro aspecto observado nesse trabalho é a importância da cobertura uniforme da calda sobre a folha do milho e a persistência de resíduos do extrato de nim após um determinado período. Visualmente, constatou-se que mesmo em uma pulverização criteriosamente bem feita, a calda-inseticida não é uniformemente distribuída sobre as folhas e, praticamente, não é depositada na parte ventral das folhas.

Isso pode ter contribuído para a diferença na eficácia de controle entre as folhas pulverizadas e submersas, desde que a mesma concentração foi utilizada para ambos os tratamentos. SCHMUTTERER (1990) relatou que o efeito residual dos extratos de nim é de cinco a sete dias, necessitando repetir a aplicação de acordo com o estádio fenológico da cultura e com o ciclo da espécie. 
Ainda segundo o autor, os insetos são capazes de diferenciar partes da planta tratada e não tratada, indicando que as pulverizações de inseticidas à base de nim devem ser realizadas cuidadosamente, utilizando alto volume e técnicas que garantam uma distribuição uniforme da calda sobre as partes das plantas a serem protegidas.

Em condições ideais para o desenvolvimento da planta, novas folhas não tratadas tornam-se disponíveis para a lagarta em curto período de tempo. Nesse caso, a lagarta pode voltar a alimentar em partes não protegidas da planta e acarretar danos ao milho.

\section{CONCLUSÕES}

1. Folhas de milho submergidas ou pulverizadas com o extrato aquoso de folhas de nim ingeridas pelas lagartas de $S$. frugiperda causam elevada mortalidade e prejudicam seu desenvolvimento.

2. O espalhante adesivo e o óleo de soja misturado ao extrato melhoram a eficiência de controle de lagartas por pulverização aos 10 dias de idade.

3. Não foi observado efeito de contato direto do extrato aquoso de nim sobre o desenvolvimento e a mortalidade de lagartas de S. frugiperda.

\section{REFERÊNCIAS BIBLIOGRÁFICAS}

ABBOTT, W.S. A method of computing the effectiveness of an insecticide. Journal of Economic Entomology, College Park, v.18, p.265-266, 1925.

ADEL, M.M.; SEHNAL, F. Azadirachtin potentiates the action of ecdsysteroid agonist RH-2485 in Spodoptera littoralis. Journal of Insect Physiology, Oxford, v.46, p.267-274, 2000.

BLANEY, W.M.; SIMMONDS, M.S.J.; LEY, W.V.; ANDERSON, J.C.; TOOGOOD, P.L. Antifeedant effects of azadirachtin and structurally related compounds on lepidopterous larvae. Entomologia Experimentalis et Applicata, Dordrecht, v.55, p.149$160,1990$.

BRUNETON, J. Pharmacognosy, phytochemistry, medicinal plants. Andover: Intercept/ Paris: Lavoisier, 1995. 915p.

CRUZ, I.; OLIVEIRA, L.J.; OLIVEIRA, A.C.; VASCONCELOS, C.A. Efeito do nível de saturação de alumínio em solo ácido sobre os danos de Spodoptera frugiperda (J. E. Smith) em milho. Anais da Sociedade Entomológica do Brasil, Londrina, v.25, p.293297, 1996.

MAREDIA, K.M.; SEGURA, O.L.; MIHM, J.A. Effects of neem, Azadirachta indica, on six species of maize insect pests. Tropical Pest Management, London, v.38, n. 2, p.190-195, 1992.
MARTINEZ, S.S.; EMDEN, H.F.van. Growth disruption, abnormalities and mortality of Spodoptera littoralis (Boisduval)(Lepidoptera: Noctuidae) caused by Azadirachtin. Neotropical Entomology, Londrina, v.30, p.113-125, 2001.

MORDUE, A.J.; BLACKWELL, A. Azadirachtin: An Update. Journal of Insect Physiology, Oxford, v.39, p.903-924, 1993.

MORDUE, A.J.; NISBET, A. Azadirachtin from the Neem tree Azadirachta indica: its actions against insects. Anais da Sociedade Entomológica do Brasil, Londrina, v.29, p.615-632, 2000.

MORDUE, A.J.; SIMMONDS, M.S.J.; LEY, S.V.; BLANEY, W.M.; MORDUE, W.; NASIRUDDIN, M.; NISBET, A.J. Actions of azadirachtin, a plant allelochemical, against insects. Pesticide Science, Oxford, v.54, p.277-284, 1998.

NATIONAL RESEARCH COUNCIL. Neem: a tree for solving global problems. Washington: National Academy Press, 1992. $139 \mathrm{p}$.

NEVES, B.P.; NOGUEIRA, J.C.M. Cultivo e utilização do nim indiano (Azadirachta indica A. Juss.). Goiânia: EMBRAPA, CNPAF; APA, 1996.32p.(Circular Técnica, 28).

PEREZ, G.; PADRON, R.; SOTO, R.; BERTSCH, F. Efecto de tres plaguicidas naturales derivados del nim sobre el combate de plagas en col y maiz en el campo y en Vigna unguiculata en almacenamiento. Agronomia-Costarricense, Cienfuegos, v.21, p.259-266, 1998.

SCHUMUTTERER, H. Potential of azadirachtin-containing pesticides for integrated pest control in developing and industrialized countries. Journal of Insect Physiology, Oxford, v.34, p.713-719, 1988.

SCHUMUTTERER, H. Properties and potential of natural pesticides from the neem tree, Azadirachta indica. Annual Review of Entomology, Palo Alto, v.35, p.271-297, 1990.

SIMMONDS, M.S.J. Molecular- and chemo-systematics: do they have a role in agrochemical discovery? Crop Protection, Oxford, v.19, p.591-596, 2000

TORRES, A.L.; BARROS, R.; OLIVEIRA, J.V.de. Efeito de extratos aquosos de plantas no desenvolvimento de Plutella xylostella (L.)(Lepidoptera: Plutellidae). Neotropical Entomology, Londrina, v.30, p.151-156, 2001.

TRINDADE, R.C.P.; MARQUES, I.M.R.;XAVIER, H.S.; OLIVEIRA, J.V.de. Extrato metanólico da amêndoa da semente de nim e a mortalidade de ovos e lagartas da traça-do-tomateiro. Scientia Agricola, Piracicaba, v.57, p.407-414, 2000.

VIÑUELA, E.; ADÁN, A.; SMAGGHE, G.; GONZÁLEZ, M.; MEDINA, M.P.; BUDIA, F; VOGT, H.; ESTAL, P.Laboratory effects of ingestion of azadirachtin by two pests (Ceratitis capitata and Spodoptera exigua) and three natural enemies (Chrysoperla carnea, Opius concolor and Podisus maculiventris). Biocontrol Science and Techonology, Oxford, v.10, p.165-177, 2000.

WILLIAMS, W.P.; DAVIS, F.M. Response of corn to artificial infestation with fall armyworm and southwestern corn borer larvae. Southwestern Entomologist, Dallas, v.15, p.163-166, 1990. 